\title{
Comparative Analysis Of Bandwidth Enhancement Of Microstrip Patch Antennas Using Various Geometries
}

\author{
S.S.Yavalkar ${ }^{1}$, R.T.Dahatonde ${ }^{2}$, Dr.S.S.Rathod ${ }^{3}$, Dr.S.B.Deosrkar ${ }^{4}$ \\ ${ }^{I}$ Student, Sardar Patel Inst. Of Technology, Mumbai, India \\ ${ }^{2}$ Electrical Dept., S.P.C E., Mumbai, India \\ ${ }^{3}$ HOD,Electronics Dept., S.P.I.T., Mumbai, India \\ ${ }^{4}$ Principal, VPCOE, Baramati, India
}

\begin{abstract}
This paper discusses the comparative study of bandwidth $(B W)$ enhancement technique of Microstrip Patch Antenna (MSA) using various geometries. A simple Rectangular Microstrip Patch Antenna (RMSA) was designed and simulated using Zeland's Method of Moment (MoM) based EM simulation package IE3D and its various parameters such as return loss, VSWR and input impédance were obtained. Then shape of this RMSA was modified by cutting various slots in it at appropriate positions. Increase in operational BW of MSA was observed.
\end{abstract}

Keywords-Rectangular Micro strip Antenna, Modified Shapes, BW Enhancement, Antenna Design.

\section{INTRODUCTION}

Physically, MSA consists of a metallic radiating patch backed up by a dielectric substrate and a ground plane below that. These days, MSAs are widely used in many applications due to their inherent advantages such as low profile, lightweight, planer configuration and ease of fabrication. However, main limitation of MSAs is their inherently narrow $\mathrm{BW}$, typically about $5 \%$ relative to resonant frequency [1].

Most of the Wireless communication applications need antenna with broad BW. Therefore, most of the recent research activities in MSA are aimed towards development of MSAs with wide impedance BW without sacrificing return loss of the antenna. Many such techniques are proposed in literature. Most of these techniques are summarized in [2].

However, techniques such as use of an thick substrate also introduces a large inductance due to increase length of the probe feed, resulting in a maximum BW of less than $10 \%$ of the resonant frequency. Also, though the designs consisting of stacked patches yields higher BWs (10\% to $20 \%$ of the resonant frequency); these designs are complex for fabrication. BW of MSA can also be increased by cutting a resonant slot inside the patch or by using multi resonator gap coupled and stacked configurations [2]. In this paper, these techniques are developed to enhance BW of RMSA. A comparative analysis of the various geometries of MSA obtained by cutting slots inside the radiating patch indicate considerable improvement in BW without much sacrifice on other performance parameters of MSA such as return loss, VSWR and its input impedance.

\section{A. Design of RMSA}

\section{Design Of Patch Antenna}

According to Transmission line model, MSA is represented as two slots separated by a transmission line. The Microstrip separates two dielectrics, i.e. air and substrate. Hence most of the electric field lines reside inside the substrate and some extend to air. This transmission line cannot support pure TEM mode of propagation since the phase velocities would be different in air and the substrate. Hence, effective dielectric constant must be obtained in order to account for fringing fields. The value of effective dielectric constant is less than dielectric constant of the substrate, because the fringing fields around the periphery of the patch are not confined in the dielectric substrate, but are also spread in the air. The value of this effective dielectric constant is given by [4];

$$
\varepsilon_{\text {eff }}=\frac{\left(\varepsilon_{r}+1\right)}{2}+\frac{\left(\varepsilon_{r}-1\right)}{2}\left[1+\frac{12 h}{W}\right]^{-\frac{1}{2}}
$$

Where in $\varepsilon_{\text {eff }}$ is effective dielectric constant and $\varepsilon_{r}\left(\mathrm{Fm}^{-1}\right), h(\mathrm{~mm}), W(\mathrm{~mm})$ are represents dielectric constant, height and width of the substrate respectively.

For RMSA to be an effective radiator, $\mathrm{W}$ should be taken equal to a half wavelength corresponding to the two dielectric mediums (i.e. substrate and air) [1]. 


$$
W=\frac{c}{2 f_{0} \sqrt{\frac{\varepsilon_{r}+1}{2}}}
$$

Where, $\mathrm{c}$ is velocity of light in free space.

The fringing fields along the width can be modeled as radiating slots increasing electrical length of patch than physical length. This increase in length is given as;

$$
\Delta L=0.412 h\left[\frac{\left(\varepsilon_{\text {eff }}+0.3\right)\left(\frac{W}{h}+0.264\right)}{\left(\varepsilon_{\text {eff }}-0.258\right)\left(\frac{W}{h}+0.813\right)}\right]
$$

Thus at resonance frequency, effective length of the patch is;

$$
L_{e}=L+2 \Delta L
$$

From these equations, dimensions were obtained for $2.45 \mathrm{GHz}$ of ISM band, The length and width of the RMSA was found to be $40 \mathrm{~mm}$ and $30 \mathrm{~mm}$, respectively.

The geometry of this RMSA is shown in Fig.1. This design was simulated using Zeland's MoM based EM Simulation Package, IE3D. For simulation purpose FR4 substrate with dielectric constant of 4.47 with thickness of $1.6 \mathrm{~mm}$ was considered. The patch was fed by a $50 \Omega$ coaxial feed line.

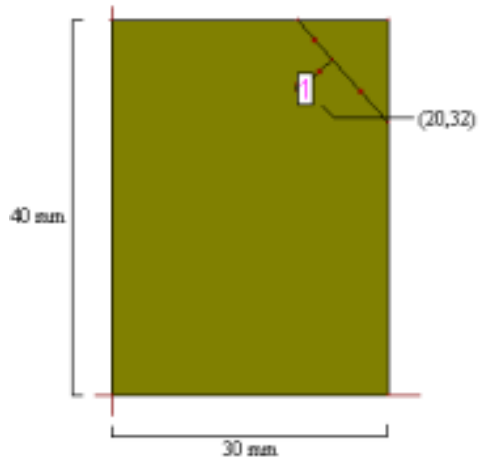

Results are discussed in Results and Discussion.

Fig. 1. Geometry of RMSA

\section{B. Modified RMSA with One Slot}

BW of MSA can be increased by cutting resonant slots inside the patch. These slots can be either half wave or quarter wave in length. The RMSA discussed in previous section was modified and a slot is introduced at its non-radiating edge as shown in Fig.2. Keeping the substrate parameters same as that for RMSA previously but feed point is changed for optimization purpose, this modified design was simulated using IE3D.

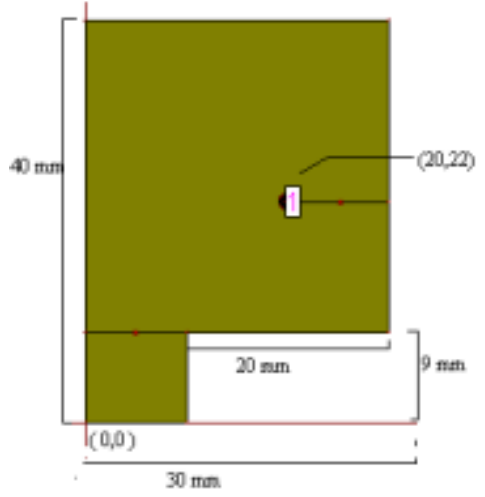

Fig.2. Geometry of RMSA with one slot.

\section{Modified RMSA with Two Slots}

The RMSA design given in previous section is again modified and one more slot with same dimensions is introduced at opposite non-resonant edge of the previous geometry having one slot, for enhancement of the BW. Fig. 3 shows the modified geometry of the RMSA followed by its results in Fig.4. 


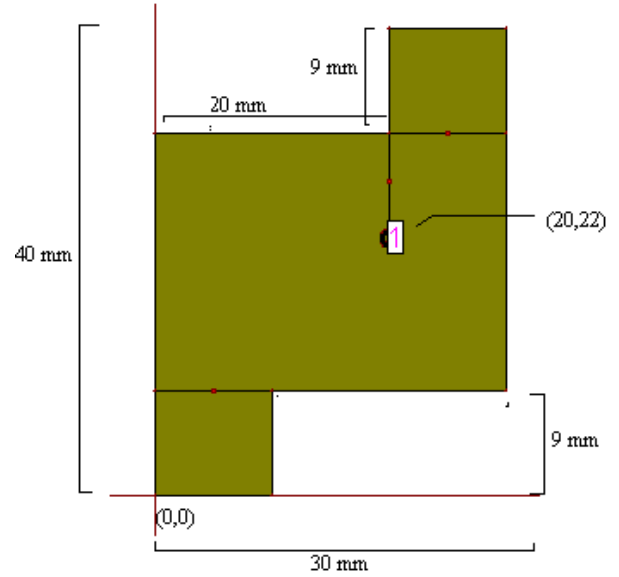

Fig.3 Geometry of RMSA with two slots

III. RESULTS AND DisCUSSION

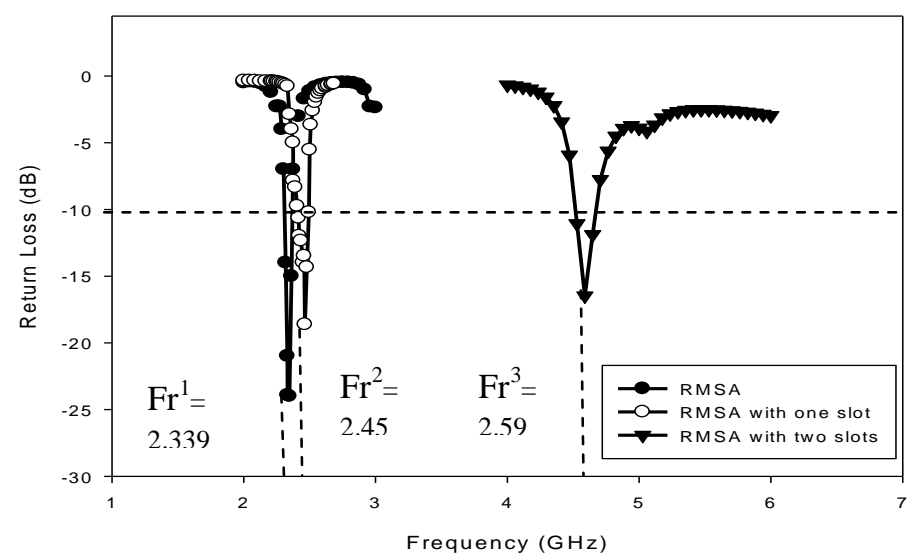

Fig.4. Comparison of Return Loss

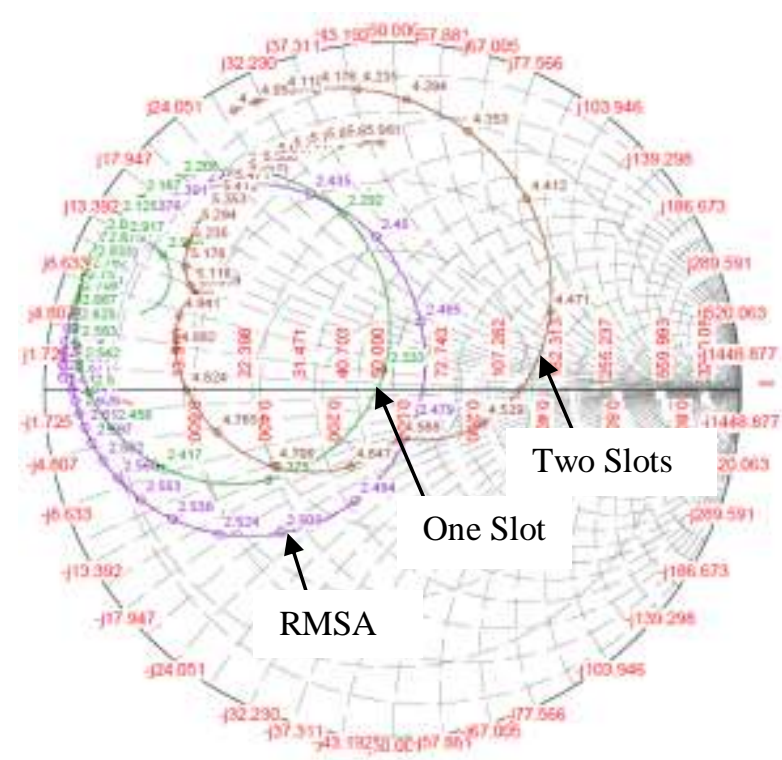

Fig.5 Comparison of input impedance in Smith Chart

Fig. 4 and 5 shows the comparison of return loss (RL) and smith chart resp. for all the geometries discussed before. Return loss (RL) below $-10 \mathrm{~dB}$ (which is corresponds to VSWR 2) is considered good for wireless communication so we draw a dotted line at $-10 \mathrm{~dB}$ in Fig.4 Frequency range below $-10 \mathrm{~dB}$ is BW.

Table 1 shows the return loss, resonance frequency and impedance of patch antenna discussed above. 


\begin{tabular}{|l|l|l|l|l|}
\hline Shape & $\begin{array}{l}\text { BW } \\
(\mathrm{MHz})\end{array}$ & $\begin{array}{l}\text { Return Loss } \\
(\mathrm{dB})\end{array}$ & $\begin{array}{l}\text { Resonance } \\
\text { Frequency fr } \\
\text { (GHz) } \\
\mathbf{i = ~ 1 , 2 , 3}\end{array}$ & $\begin{array}{l}\text { Impedance } \\
(\Omega)\end{array}$ \\
\hline RMSA & 35 & -25 & 2.339 & 49 \\
\hline $\begin{array}{l}\text { RMSA with } \\
\text { one slot }\end{array}$ & 50 & -20 & 2.459 & 45 \\
\hline $\begin{array}{l}\text { RMSA with } \\
\text { two slot }\end{array}$ & 150 & -15 & 4.59 & 52 \\
\hline
\end{tabular}

Table 1. Comparison of Geometries

The BW of RMSA is $35 \mathrm{MHz}(1.45 \%)$. When two parallel slots are introduced in the non-radiating side of same patch then BW is increased to $150 \mathrm{MHz}(3.26 \%)$.

In RMSA current distribution at its edges is always comparatively minimum for TM10 mod. We choose the position of slot such that they change the path of current. That is the reason why resonance frequency for RMSA with slot is different. Because of slot the surface current path decreases and as a result resonant frequency increases. This effect can be observed in Fig.4.

Also when slot gets introduced in the patch, another mode gets introduced near its fundamental mode and so BW gets increases near its fundamental mode and so BW gets increases. This effect can be seen from Fig. 4

We uses $50 \Omega$ coaxial connecter for feeding the antenna. For maximum power to be transferred as per maximum power transfer theorem the impedance should be $50 \Omega$ for resonance frequency. From Fig. 5 we can observe that the impedance is nearly $50 \Omega$ for resonance frequencies of all geometries.

\section{CONCLUSION}

A comparative study of effect of shape on bandwidth and return loss of patch antenna is presented in this paper. It was observed that, only dielectric constant and thickness of patch antenna does not affects the BW but also its shape plays an important factor to enhance the BW. The resonance frequency gets shifted due to change in current path occurs due to slots introduction in path. So, cutting the slots in the patch affects the resonance frequency of higher modes and hence it realizes higher BW.

\section{REFERENCES}

[1] Kumar G. and Ray K.P.,Broadband Microstrip Antenna,Artech House Publication,pp.1-291,2003.

[2] Kin Lu Wong, Compact and Broadband Microstrip Antennas,John Wiley \& Sons,pp.45-79,2002.

[3] Bhartia P., Millimeter-Wave Microstrip and Printed Circuit Antennas, Artech House,pp.1-10,1991.

[4] Balanis C.A., Antenna Theory Analysis and Design,2nd Edi.,John Wiley \& Sons,pp.730-750,1997.

[5] Deshmukh A.A., Perulekar M., Kadam S. and RAY K.P, Broadband Proximity Fed Modified E-Shape Microstrip Antenna,” NCC Conf.,pp.1-5,2011. 